\title{
Evaluación multicriterio de los recursos turísticos en la Parroquia Uzhcurrumi, Canton Pasaje, Provincia de El Oro
}

\section{Multicriteria evaluation of the tourist resources in Uzhcurrumi Parish, Pasaje Canton, Province of El Oro}

David Cartuche P.1, Juleysi Romero D.2, Yajaira Romero D.3

Resumen: El potencial turístico de un territorio depende de la valoración que se realiza a sus recursos. La provincia de El Oro, situada en la región litoral del Ecuador, cuenta con una gran biodiversidad y una belleza paisajística que la hace idónea para el desarrollo de actividades turísticas. El objetivo de este trabajo fue realizar un análisis de las posibilidades de aprovechamiento de los recursos turísticos de la parroquia Uzhcurrumi, cantón Pasaje, provincia del Oro, Ecuador. Como metodología se consideró el inventario de 14 recursos existentes y la evaluación cuantitativa con base en las técnicas de evaluación multicriterio discreta (EMC) partiendo de la definición de criterios intrínsecos y extrínsecos para cada tipo de recurso. Posteriormente se determinaron los atributos de análisis y se construyó la matriz de decisión. La aplicación de la combinación lineal ponderada condujo a la jerarquización final de los recursos turísticos. Los resultados de este trabajo revelan que el Balneario de Chillayacu y Las Cavernas de Chillayacu son los recursos de mayor calificación, sobre los que se propone iniciar el desarrollo de potenciales productos turísticos. Una importante implicancia es que la evaluación de los recursos turísticos es una herramienta valiosa para orientar la toma de decisiones en los procesos de planificación y desarrollo turísticos.

Palabras clave: Potencial turístico, valoración, biodiversidad, evaluación multicriterio, recursos turísticos.
Abstract: The tourist potential of a territory depends on the assessment of its resources. The province of El Oro, located in the coastal region of Ecuador, has great biodiversity and a scenic beauty that makes it ideal for tourist activities. The aim of this work was to analyze the possibilities of utilizing the tourist resources of Uzhcurrumi Parish, Pasaje Canton, Province of El Oro, Ecuador. As a methodology, the inventory of 14 existing resources was considered, as well as a quantitative evaluation based on the techniques of multicriteria evaluation tools (MET), starting with the definition of intrinsic and extrinsic criteria for each resource type. Then, the study attributes were determined and the decision matrix was constructed. The application of the weighted linear combination led to the final ranking of the tourist resources. The results reveal that the Chillayacu Spa and the Chillayacu Caverns are the best qualified resources, so it is suggested that they be the starting point for development of potential tourist products. An important implication is that the evaluation of tourist resources is a valuable tool to guide decision-making in tourist planning and development processes.

Key words: Tourist potential, assessment, biodiversity, multicriteria evaluation, tourist resources.

(Presentado: 10 de julio de 2018. Aceptado: 10 de septiembre de 2018)

\footnotetext{
${ }^{1}$ Master en Gestión y Promoción del Desarrollo Local por la Universidad de Valencia-España. Docente en la Universidad Técnica E-mail: dcartuchequtmachala.edu.ec

${ }^{2}$ Administración de Hotelería y Turismo. Universidad Técnica de Machala, Ecuador. E-mail: jtromero_estąmachala.edu.ec

${ }^{3}$ Administración de Hotelería y Turismo. Universidad Técnica de Machala, Ecuador. E-mail: ygromero_estQqutmachala.edu.ec
} 


\section{ITRODUCCIÓN}

La provincia de El Oro cuenta con una gran biodiversidad y una belleza paisajística que la hace idónea para el desarrollo de actividades turísticas, la zona de estudio es la parroquia Uzhcurrumi, del Catón Pasaje; la cual posee áreas naturales que propician al desarrollo de diferentes actividades turísticas.

Uzhcurrumi es una parroquia rural considerada como un poblado preincaico que posee hermosos paisajes, tiene una extensión de $35 \mathrm{~km} 2$, sentada a orillas del Río Jubones y rodeada por montañas, formando un paradisiaco Valle. Es un importante centro de producción ganadera y agrícola, de transcendental importancia histórica, puesto que hasta los años ' 80 fue un significativo centro de comercio entre la Provincia de El Oro y la Provincia del Azuay. Es una zona privilegiada por su riqueza natural y arqueológica, cuenta con recursos turísticos naturales como las Cascadas de Portillo, Balneario de Quera, las encantadas Cavernas de Chillayacu, al igual que lugares históricos como el Puente Histórico que limita la provincia de El Oro con la Provincia del Azuay, el Templete a los Héroes de Porotillo y los petroglifos ubicados en el Sitio Chillayacu. Por la limitada promoción turística, y a partir de las visitas de exploración realizadas en la Parroquia, se ha considerado pertinente realizar una valoración del potencial turístico de los recursos naturales y culturales, susceptibles a convertirse en productos turísticos, para ampliar y diversificar la oferta turística de la parroquia y la provincia; para lo cual fue necesaria la identificación de los recursos turísticos a través de un inventario de atractivos turísticos y la aplicación de la metodología de evaluación multicriterio. El inventario de atractivos y recursos turísticos "constituye un registro y valoración del estado de todos los elementos que por sus cualidades naturales y culturales pueden constituir una motivación para el turista" (Font, Cuétara, \& Castanedo, 2013). Es la materia prima, sobre la cual han de desarrollarse actividades turísticas. Convirtiéndose en un "elemento indispensable para determinar el tipo y la ubicación de las inversiones que han de reforzar la planta turística del lugar, ya que en función del inventario es posible definir todos los espacios turísticos" (Cárdenas, 2012), que formarán parte de la oferta turística de la comunidad.

El presente trabajo de investigación tiene como finalidad identificar los recursos turísticos de la parroquia Uzhcurrumi, cantón Pasaje, con mayor potencial sobre los cuales se puedan desarrollar productos turísticos, mediante la evaluación multicriterio y la suma lineal ponderada.

\section{MARCO TEÓRICO}

El potencial turístico de un territorio depende de la valoración que se realiza a sus recursos. Bote (2002) y Godfrey \& Clarke (2000) explican que una evaluación de recursos turísticos comprende básicamente dos fases: la elabocación de un invetario de recursos potenciales, y la evaluación de los recursos inventariados. La cual puede desarrollarse desde una perspectiva cuantitativa como lo manifesta Bote, o desde una perpectiva cualitativa como lo establece Godfrey y Clarke. Autores como Beta y Vega (2005), concuerdan en que un inventario de recursos turísticos es como un catálogo en el que se muestraN lugares y estableciemientos localizados en una determinada área geográfica, clasificando los recursos según su calidad y el grado de interés que provocan en el visitante. Explican que para ello se debe seguir un proceso, en primera instancia se debe realizar una investigación blibliográfica y documental que permita crear un listado con los sitios de interés, posteriormente se debe llenar las ficha de registro, las cuales contienen distintas variables de información como: nombre, ubicación, accesibilidad, dimensiones, distancias, conectividad, estado de conservación, seguridad y algunos más específicos como el tipo del atractivo (clasificación de atrativos turísticos) y las actividades que se pueden desarrollar. Los autores de inventarios sugieren incluir una fotografía del recurso en cada ficha de registro y un mapa aéreo o topográfico que permita observar la ubicación exacta de cada sitio de interés. Oscos, (1990) menciona que el levantamiento de la información debe ser completamente objetiva, tal y como se presenta, sin ningún tipo de valoraciones o creencias. Por otro lado, las fichas deben ser llenadas en el momento, para evitar posibles olvidos. Zamorano (2002) divide al inventario de atractivos en reales y potenciales, y considerar tres matrices: de "funcionalidad", incluyendo elementos como la movilidad, forma de desplazamiento, forma de recorrido, distancia, gradiente o pendiente, ancho de senderos; el de "interpretación estética", abarcando las oportunidades interpretativas, material (puentes, cabañas, etc.); y de "integración", que señala las unidades o puntos de interés, tradiciones, manifestaciones, flora y fauna, y el panorama general de los atractivos. SECTUR (2004), sugiere además, que se haga uso de la concepción que se tiene de cada recurso por parte de la población local mediante tres técnicas integradas: elaboración de dibujos por parte de los habitantes locales, relatos hechos por los pobladores sobre su modo de vivir y descripción de los recursos con los que cuentan, y por último la observación de la conducta de la 
población, obteniendo datos acerca de qué se hace, quién lo lleva a cabo, cómo lo realiza y cuándo sucede; esta última técnica es aplicada únicamente para el inventario de recursos culturales.

En Ecuador la metodología para la evaluación y jerarquización de atractivos, establece los lineamientos técnicos para la identificación, clasificación y valoración de los atractivos con mejores condiciones para el desarrollo de productos turísticos. Esta metodología no mide la atractividad en sí, referido a qué tan atractivo es un sitio, sino que jerarquiza los atractivos que presentan las mejores condiciones para el desarrollo de productos turísticos. El procedimiento se realizará en dos etapas: la primera permitirá el levantamiento, registro, jerarquización y espacialización de atractivos turísticos; y la segunda será la identificación de atractivos, análisis geográfico y tipificación de espacios turísticos. (MINTUR, 2017). Herrera, Crespo, Zambrano y Cadena, (2014), desarrollan un Análisis Jerárquico Multicriterio, para la valoración y jerarquización de recursos turísticos en el Cantón Rumiñahui (Ecuador). Identifican cinco sitios relevantes, dos son patrimonio cultural intangible, uno patrimonio cultural tangible y otro patrimonio natural. Sugieren que las actividades de gestión turística del cantón se deben orientar a fortalecer y promover la segmentación del mercado. Además, que los sistemas de valor en torno a la actividad turística se deben orientar a la oferta y creación de experiencias únicas que responden a necesidades concretas de la demanda. Por su parte, Morales (2013), realizó el inventario turístico municipal Querétaro (México), recolectando la información turística disponible para constituir un área turística. La metodología utilizada fue multicriterio para la Jerarquización de los atractivos y construcción de estadísticas. Las conclusiones sugieren integrar tres sitios al producto de turismo cultural mediante un discurso histórico, económico y arquitectónico. Sugieren que las fachadas de las casas y comercios deben ser restauradas y conservadas mediante un programa de mejoramiento de la imagen urbana que respete las técnicas constructivas del lugar y que el centro histórico de Querétaro sirva para visitantes que deseen hacer recorridos alternativos

\section{METODOLOGÍA}

\section{Ubicación geográfica}

La Parroquia Uzhcurrumi está ubicada al sur de la costa ecuatoriana, en la parte norte de la Provincia de El Oro y el Cantón Pasaje, a 150 msnm. Limita al norte con el cantón Pucará, al sur con la parroquia Casacay, al este, con el Cantón Chilla y al oeste con el Río Jubones.

\section{Figura 1: Uicación geográfica.}

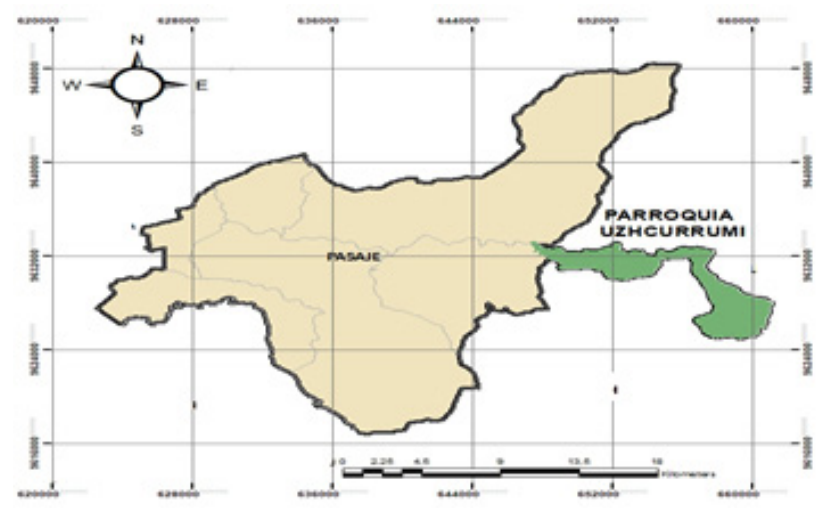

\section{Inventario de atractivos turísticos}

Para seleccionar los recursos turísticos de un territorio es necesario "identificar los lugares de interés turístico, inventariarlos y tipificarlos, para posteriormente seleccionar aquellos que se consideren prioritarios" (Blanco , Vázquez, Reyes, \& Genet, 2015); para lo cual se utilizan una serie de criterios y atributos, que dependerán básicamente de las características específicas de cada territorio, permitiendo obtener la información relevante que permita desarrollar proyectos y programas de carácter público y privado.

La elaboración de un inventario que integre la información más representativa y relevante de los recursos potencialmente turísticos de una localidad es un trabajo transcendental para la evaluación y estructuración de los productos turísticos (Enríquez, 2010). Este debe contemplar los elementos esenciales que se requiere para levantar la información, teniendo en cuenta el tipo de recurso y los datos relevantes que se pretende obtener del mismo. En función de ello es que se adoptó la metodología propuesta por el Ministerio de Turismo en el 2017, para realizar el inventario de atractivos turísticos. Como resultado del inventario realizado en la Parroquia Uzhcurrumi, se ha identificado 14 recursos turísticos potenciales (Tabla 1). 
Tabla 1. Recursos turísticos de la Parroquia Uzhcurrumi.

\begin{tabular}{ll}
\hline Cavernas de Chillayacu & \\
\hline Categoría & Atractivo Natural \\
Tipo & Fenómenos Geológicos \\
Subtipo & Cueva o Caverna \\
Jerarquía & II \\
Monumento Héroes del 41 & \\
\hline Categoría & Manifestaciones Culturales \\
Tipo & Arquitectura \\
Subtipo & Monumento \\
Jerarquía & I \\
lglesia Uzhcurrumi & \\
\hline Categoría & Manifestación Cultural \\
Tipo & Arquitectura \\
Subtipo & Área Histórica \\
Jerarquía & I \\
La Tarabita de Leo & \\
\hline Categoría & Manifestaciones Culturales \\
Tipo & Acontecimientos Programados \\
Subtipo & Eventos Deportivos \\
Jerarquía & II \\
Balneario de Quera & \\
\hline Categoría & Atractivos Naturales \\
Tipo & Ríos \\
Subtipo & Rio \\
Jerarquía & I \\
Balneario Chillayacu & \\
\hline Categoría & Atractivos naturales \\
Tipo & Ríos \\
Subtipo & Río \\
Jerarquía & III \\
Cascada Porotillo & \\
\hline Categoría & Atractivos Naturales \\
Tipo & Ríos \\
Subtipo & I ascadas \\
Jerarquía & \\
\hline EunteEborion & \\
\hline
\end{tabular}

Fuente: Elaboración propia con base en los

levantamientos de las fichas de campo.

Evaluación Multicriterio (MCE).

La evaluación multicriterio comprende "un conjunto de técnicas que permiten evaluar diversas alternativas de elección a la luz de múltiples criterios y prioridades" (Ocaña \& Galacho , 2002 Pág.240). Al evaluarse una serie de alternativas que satisfacen uno o varios objetivos, permite que las decisiones sean tomadas de forma multidimensio-

\begin{tabular}{|c|c|}
\hline \multicolumn{2}{|l|}{ Artesanías } \\
\hline Categoría & Manifestaciones Culturales \\
\hline Tipo & Folcklore \\
\hline Subtipo & Artesanías y Artes \\
\hline Jerarquía & I \\
\hline \multicolumn{2}{|c|}{ Museo Parroquial Uzhcurrumi } \\
\hline Categoría & Manifestaciones Culturales \\
\hline Tipo & Arquitectura \\
\hline Subtipo & Museos \\
\hline Jerarquía & I \\
\hline \multicolumn{2}{|c|}{ Parque Central Uzhcurrumi } \\
\hline Categoría & Manifestación Cultural \\
\hline Tipo & Arquitectura \\
\hline Subtipo & Espacio Público \\
\hline Jerarquía & I \\
\hline \multicolumn{2}{|c|}{ Petroglifos de Chillayacu } \\
\hline Categoría & Manifestaciones Culturales \\
\hline Tipo & Ruinas \\
\hline Subtipo & Petroglifos \\
\hline Jerarquía & 1 \\
\hline \multicolumn{2}{|c|}{ Puente Histórico } \\
\hline Categoría & Manifestación Cultural \\
\hline Tipo & Arquitectura \\
\hline Subtipo & Área Histórica \\
\hline Jerarquía & I \\
\hline \multicolumn{2}{|c|}{ Fiestas Religiosas } \\
\hline Categoría & Manifestación Cultural \\
\hline Tipo & Folcklore \\
\hline Subtipo & Fiestas Religiosas \\
\hline Jerarquía & I \\
\hline \multicolumn{2}{|c|}{ Fiestas Parroquiales } \\
\hline Categoría & Manifestación Cultural \\
\hline Tipo & Folcklore \\
\hline Subtipo & Fiestas \\
\hline Jerarquía & I \\
\hline
\end{tabular}

nal. Los modelos multiobjetivos y los análisis multicriterio, ofrecen la posibilidad de obtener un estudio equilibrado de todos los atributos establecidos y de todas las facetas de los problemas de planificación (Laguna \& Bravo, 2001). Se basa en la construcción de una matriz que "refleja las características de un conjunto dado de alternativas de elec- 
ción a partir de una serie de criterios” (Mikery \& Pérez, 2017 Pág.1732). La matriz "expresa las cualidades (valor numérico o simbólico) de la alternativa o unidad de observación i con respecto a los $n$ atributos considerados (Barba \& Pome- rol, 1997). El conjunto de alternativas se caracterizan por una serie de atributos, que al añadirles información, relativa a las preferencias del decisor, se consideran criterios. La Tabla 2 presenta los componentes básicos de la EMC.

\section{Tabla 2. Descripción de los componentes para la valoración de los recursos turísticos.}

\begin{tabular}{ll}
\hline Componente & Descripción \\
\hline Identificación del Conjunto de & Dependerá del objetivo, del grado del problema (alternativas de decisión) \\
alternativas posibles. & El concepto de atributos así como de objetivos se incluye en el criterio de \\
Definición del conjunto de atributos & evaluación. La cuantificación de un objetivo se realiza mediante la \\
& adopción de una escala que permita medir el nivel de logro (atributo)
\end{tabular}

Obtención de atributos (normalizados)

Generalmente la información geográfica se expresa en diversas escalas (de intervalo, de razón, nominal, ordinal), por tano deben ser transformadas en una escala común, a través de procesos de conversión. La matriz de decisión permite expresar las variables numéricamente y que se y que tengan una misma escala de medida, para ello es preciso aplicar un proceso de normalización.

Combinación de los atributos normalizados y ponderados utilizando una regla de decisión para obtener el puntaje total para alternativa.
La Sumatoria Lineal Ponderada, es uno de los métodos compensatorios más utilizados. Cuyo método contempla que el rendimiento de una alternativa en un criterio específico, puede ser compensado por su rendimiento en otro criterio.

$$
r_{i}=\sum_{j=1}^{n} W_{j} V_{i j}
$$

Siendo:

$\boldsymbol{r}_{i}=$ el nivel final de cumplimiento del objetivo de la alternativa $\mathbf{i}$.

$\boldsymbol{W}_{j}=$ el peso del criterio $\mathbf{j}$.

$\boldsymbol{V}_{i j}^{j}=$ el valor ponderado de alternativa i en el criterio $\mathbf{j}$.

Fuente: Elaboración a partir de Franco (2009).

\section{Evaluación de los recursos turísticos de la Parroquia Uzhcurrumi}

Considerando los componentes básicos de la Evaluación Multicriterio (EMC), descritos en la Tabla 2, se desarrolló un procedimiento que incluyó las siguientes etapas: a) definición del conjunto de atributos; b) construcción de la matriz de decisión; y c) valoración de los recursos turísticos a través del método de sumatoria lineal ponderada.

\section{Definición del conjunto de atributos}

Para la evaluación de los recursos turísticos fue necesario establecer un conjunto de criterios, para los cuales se consideraron dos tipos: intrínsecos y extrínsecos. Los intrín- secos permiten analizar las características específicas, de cada recurso de acuerdo a su naturaleza. Los extrínsecos en cambio analizan variables recreativo-turísticas en función de las condiciones de uso (Bote, 2002).

Los criterios intrínsecos hacen alusión a elementos visuales vinculados con el espacio (vegetación, extensión y transparencia de agua); características del terreno (gradiente, tipo de superficie y grado de dificultad); dimensiones y atractivos adicionales. Estos atributos han sido establecidos a partir de las recomendaciones de Zamorano (2002) y de Gutiérrez (1998). Para los criterios extrínsecos se consideraron condiciones de acceso, grado de conser- 
vación, condiciones de uso y recreación, y seguridad, establecidos en base a las recomendaciones de Zamorano (2002) y del SECTUR (2004). Ambos tipos de criterios han sido adaptados a las características de los recursos de la parroquia Uzhcurrumi. En la Tabla 3 los criterios establecidos para las dos categorías.

Tabla 3. Criterios y atributos utilizados para la evaluación de los recursos turísticos.

\section{Criterios intrínsecos}

Tipo de recurso

Criterio

a) Cobertura

Terrestres

(Cerros, barrancas, parques, etc.) b) Condiciones del terreno

b1) Gradiente

b1) Gradiente

b2) Superficie

\section{Descripción}

Abundante (A), Suficiente (S), Escasa (E)

Muy inclinado (MI), Inclinado (I), Algo inclinado (Al), Plano (P)

Pedregosa (Pe), Terracería (T), Húmeda $(\mathrm{H})$, Compacta (C), Pastizal (Pa), Otra (O)

b3) Dificultad

Alto (A), Medio (M), Bajo (B)

Acuáticos

c) Características

(corrientes de agua)
c1) Ancho
Metros

c2) Transparencia Cristalina (C), Semiturbia (S), Turbia (T)

c3) Extensión visible 1 a 3 m (A), 3 a 6 m (B), 6 a 10 m (C), Más de $10 \mathrm{~m}(\mathrm{D})$

d) Atractivo adicional

d1) Fauna Sí (S), No (N)

e1) Estado Tradicional Excelente(E), Bueno (B), Malo(M) e2) Difusión

e3) Participación

Comunitaria

f) Promoción f1) Medios de Promoción
Local (L), Regional (R), Nacional (N), Internacional (I)

Culturales

\section{Criterios extrínsecos}

Criterio g) Acceso físico

\section{Atributo}

g1) Distancia

g2) Tiempo

h1) Estado de conservación

h) Posibilidades de apreciación
h2) Calidad del entorno

h4) Fragilidad

h5) Contaminación
Descripción

\section{Kilómetros}

Minutos
$\mathrm{Si}(\mathrm{S})$, No (N)

Web (W), Televisor (T), Radio (R), Revistas (Re)

\begin{tabular}{lll}
\hline \multirow{3}{*}{$\begin{array}{ll}\text { i) Infraestructura } \\
\text { y servicios }\end{array}$} & Suficiente (S), Alguna (A), Insuficiente (I), Ninguna (N) \\
\cline { 2 - 3 } & i2) Equipamiento & Suficiente (S), Alguno (A), Insuficiente(I), Ninguno (N) \\
\cline { 2 - 3 } i3) Instalaciones recreativas & $\begin{array}{l}\text { Instalaciones recreativas (A), Elementos susceptibles } \\
\text { de recreación (M), Ningún lugar recreativo (B) }\end{array}$ \\
\cline { 2 - 3 } j) Seguridad & i4) Actividades & No. total de actividades \\
\hline
\end{tabular}




\section{Construcción de los criterios intrínsecos}

Definidas las variables y atributos a considerar y basados en la información obtenida en el campo, se obtuvo la matriz con la caracterización cualitativa o cuantitativa de cada recurso para cada uno de los criterios. La Tabla 4 muestra la caracterización de los criterios intrínsecos.

Tabla 4. Caracterización de los criterios intrínsecos de los recursos turísticos de la Parroquia Uzhcurrumi.

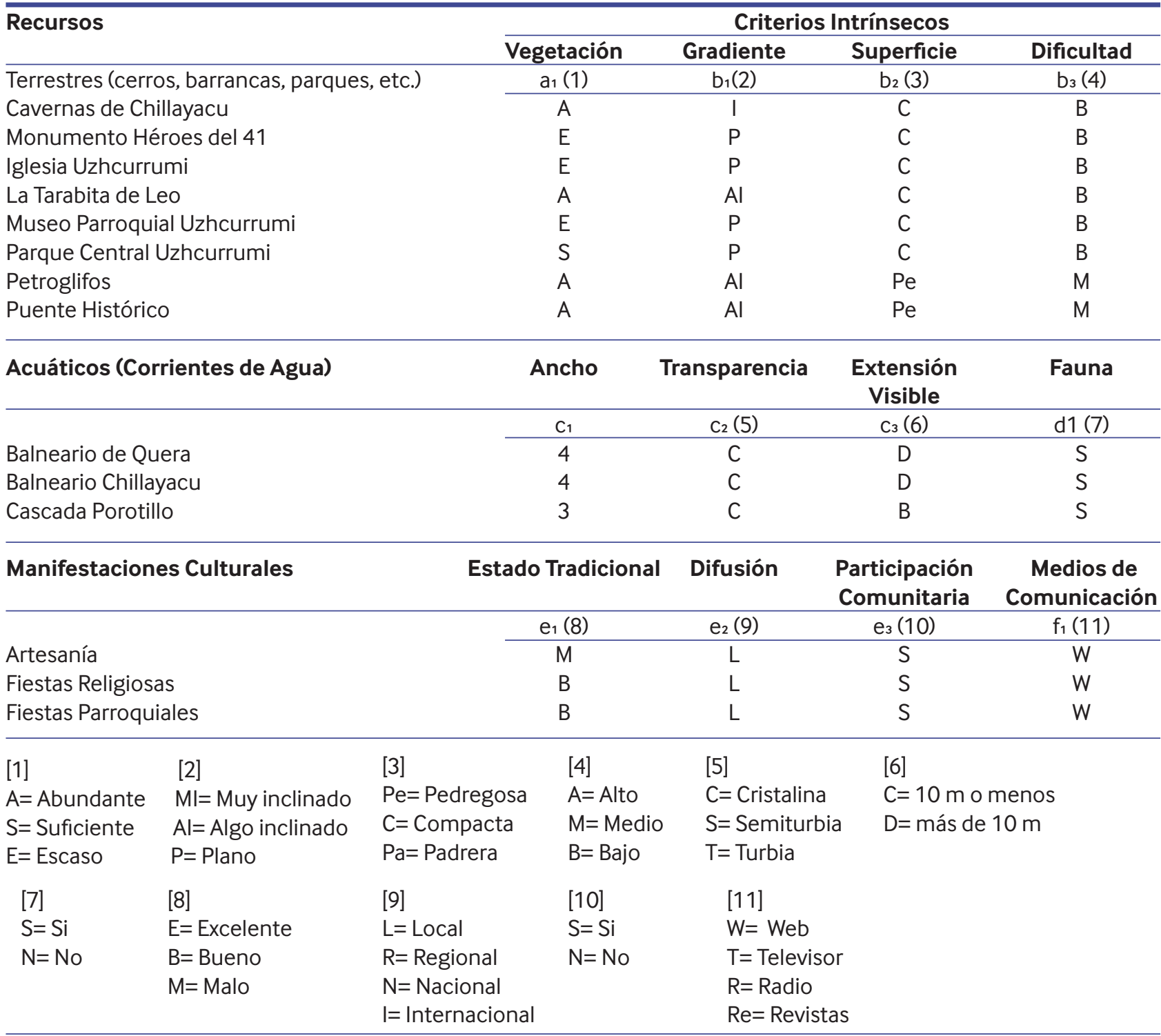

Fuente: Elaboración Propia a partir de Franco (2009). 


\section{Construcción de los criterios extrínsecos}

Del mismo modo, partiendo de las variables ya establecidas y de la información obtenida en el campo, ha sido po- sible la construcción de la matriz con los criterios extrínsecos para cada uno de los recursos de estudio. La Tabla 5 presenta la caracterización de los criterios extrínsecos.

Tabla 5. Caracterización de los criterios extrínsecos de los recursos turísticos en la Parroquia Uzhcurrumi.

\section{Criterios extrínsecos}

Recursos

\section{Acceso}

Físico

Terrestres

Cavernas de Chillayacu

Monumento Héroes del 41

g1 g2

Pos

Iglesia Uzhcurrumi

La Tarabita de Leo

Museo Parroquial Uzhcurrumi

Parque Central Uzhcurrumi

Petroglifos

20 52 h1 h2 h3

Puente Histórico

$5 \quad 20$

E $O \quad G$

h3 h4 h5

B $B$ P

B $\mathrm{S}$

15

15

$\begin{array}{lcl}1 & 15 & R\end{array}$

199

119

E O G

B

$30 \quad 70$

1420

Acuáticos

Balneario de Quera

Balneario Chillayacu

$3070 \quad E$

O G

B $\quad \mathrm{N}$

5

i1 i2

$\begin{array}{cc}\text { i3 } & \text { Seguridad } \\ \text { i4 j1 }\end{array}$

Cascada Porotillo

$20 \quad 50$

Manifestaciones culturales

Artesanía

Fiestas Religiosas

Fiestas Parroquiales

$18 \quad 25$

E

0

$B$

$\begin{array}{llllll}N & N & N & B & 1 & S\end{array}$

A $3 \quad M$

$\begin{array}{llllll}N & N & A & B & 2 & M\end{array}$

$O A$

B $\quad \mathrm{N} \quad \mathrm{S} S$

A $3 \quad M$

$\begin{array}{llllllllll}B & O & A & B & N & N & A & M & 1 & M\end{array}$

$\begin{array}{lllllllll}R & B & A & M & N & N & N & B & 2\end{array}$

Fuente: Elaboración propia a partir de Franco (2009).

\section{Obtención de la matriz de decisión}

Una vez que se realizó la tabulación de los criterios, se realizó su transformación a una escala de 1 y 10 . El valor de cada atributo hace referencia al grado n que cada atributo cumple con el objetivo de valoración, siendo 10 el valor máximo y 1 el valor mínimo, basados en esto, fue posible la construcción de la matriz. La Tabla 6 presenta la matriz de decisión. 
Tabla 6. Matriz de decisión con valores normalizados.

\begin{tabular}{|c|c|c|c|c|c|c|c|c|c|c|c|c|c|c|c|c|}
\hline \multirow{3}{*}{$\begin{array}{l}\text { Recursos } \\
\text { Terrestres }\end{array}$} & \multicolumn{16}{|c|}{ Atributos } \\
\hline & va & $\mathrm{vb}$ & $\mathrm{vb}$ & $\mathrm{vb}$ & vg & vg & $\mathrm{vh}$ & vh & vh & $\mathrm{vh}$ & $\mathrm{vh}$ & \multirow{2}{*}{ vi1 } & \multirow{2}{*}{ vi2 } & \multirow{2}{*}{ vi3 } & \multirow{2}{*}{ vi4 } & \multirow{2}{*}{ vj1 } \\
\hline & 1 & 1 & 2 & 3 & 1 & 2 & 1 & 2 & 3 & 4 & 5 & & & & & \\
\hline Cavernas de Chillayacu & 10 & 3.5 & 9 & 10 & 5.5 & 2 & 10 & 9 & 8 & 7 & 5 & 2 & 4 & 6 & 5 & 5 \\
\hline Monumento Héroes del 41 & 1 & 10 & 9 & 10 & 6 & 5 & 1 & 2.5 & 5 & 5 & 7 & 1 & 1 & 1 & 1 & 5 \\
\hline Iglesia Uzhcurrumi & 1 & 10 & 9 & 10 & 6 & 4 & 1 & 2 & 5 & 7 & 8 & 1 & 2 & 1 & 2 & 4 \\
\hline La Tarabita de Leo & 10 & 6 & 7 & 8 & 8 & 7 & 6 & 5 & 5 & 6 & 8 & 3 & 5 & 6 & 7 & 10 \\
\hline Museo Parroquial Uzhcurrumi & 1 & 10 & 9 & 10 & 8 & 6 & 3.5 & 6 & 4 & 4 & 3 & 1 & 2 & 1 & 2 & 3 \\
\hline Parque Central Uzhcurrumi & 7 & 10 & 9 & 10 & 6 & 4 & 1 & 5 & 4 & 3 & 4 & 1 & 2 & 3 & 1 & 6 \\
\hline Petroglifos & 10 & 6 & 6 & 5 & 2.5 & 1 & 5 & 5 & 3.5 & 6 & 9 & 1 & 1 & 1 & 4 & 2 \\
\hline Puente Histórico & 10 & 6 & 6 & 5 & 1 & 5 & 5 & 4.5 & 2 & 8 & 5 & 1 & 1 & 1 & 1 & 2 \\
\hline \multirow{2}{*}{ Acuáticos } & vc & vc & vc & $\mathrm{vd}$ & vg & vg & vh & $\mathrm{vh}$ & vh & $\mathrm{vh}$ & $\mathrm{vh}$ & \multirow{2}{*}{ vi1 } & \multirow{2}{*}{ vi2 } & \multirow{2}{*}{ vi3 } & \multirow{2}{*}{ vi4 } & \multirow{2}{*}{ vj1 } \\
\hline & 1 & 2 & 3 & 1 & 1 & 2 & 1 & 2 & 3 & 4 & 5 & & & & & \\
\hline Balneario de Quera & 9 & 10 & 2.5 & 10 & 2 & 1 & 10 & 5 & 5 & 5 & 1 & 1 & 5 & 5 & 10 & 5 \\
\hline Balneario Chillayacu & 9 & 10 & 1 & 10 & 5 & 4.5 & 8 & 7 & 10 & 6 & 8 & 5 & 7 & 7 & 6 & 10 \\
\hline Cascada Porotillo & 5 & 10 & 1 & 10 & 10 & 5 & 3 & 2 & 4 & 5 & 6 & 1 & 1 & 3 & 1 & 3 \\
\hline Manifestaciones & ve & ve & ve & vf & vg & vg & vh & vh & vh & vh & $\mathrm{vh}$ & \multirow{2}{*}{ vi1 } & \multirow{2}{*}{ vi2 } & \multirow{2}{*}{ vi3 } & \multirow{2}{*}{ vi4 } & \multirow{2}{*}{ vj1 } \\
\hline culturales & 1 & 2 & 3 & 1 & 1 & 2 & 1 & 2 & 3 & 4 & 5 & & & & & \\
\hline Artesanía & 1 & 1 & 10 & 10 & 1 & 1 & 3 & 3 & 4 & 3 & 8 & 1 & 1 & 1 & 1 & 5 \\
\hline Fiestas Religiosas & 5 & 1 & 10 & 10 & 1 & 1 & 5 & 6 & 2.5 & 1 & 3 & 1 & 4 & 3 & 5 & 3 \\
\hline Fiestas Parroquiales & 5 & 1 & 10 & 10 & 1 & 1 & 3 & 7 & 3 & 1 & 4 & 1 & 5 & 5 & 7 & 3 \\
\hline
\end{tabular}

Fuente: Elaboración propia a partir de Franco (2009)

\section{RESULTADOS}

Debido a que existían tres grupos distintos de criterios intrínsecos como son: los terrestres, acuáticos y manifestaciones culturales; la evaluación implicó aplicar, para cada uno de los grupos, la sumatoria lineal ponderada.
Con base en el procedimiento de combinación se obtuvo la valoración final, de cada uno de los recursos turísticos en función de su relevancia para el desarrollo turístico en la Parroquia. 
Tabla 7. Resultados obtenidos de la combinación lineal de atributos.

\begin{tabular}{lccccc}
\hline \multicolumn{1}{c}{ Recurso } & $\begin{array}{c}\text { Suma lineal } \\
\text { sin ponderación }\end{array}$ & $\begin{array}{c}\text { Suma ponderada } \\
\text { de criterios } \\
\text { extrínsecos }\end{array}$ & $\begin{array}{c}\text { Suma ponderada } \\
\text { de criterios } \\
\text { intrínsecos }\end{array}$ & Valor Final & $\begin{array}{c}\text { Orden de } \\
\text { Preferencia }\end{array}$ \\
\hline Cavernas de Chillayacu & 68.5 & 3.43 & 3.35 & 6.78 & 3 \\
Monumento Héroes del 41 & 40.5 & 2.03 & 3 & 5.03 & 8 \\
Iglesia Uzhcurrumi & 43 & 2.15 & 3 & 5.15 & 7 \\
La Tarabita de Leo & 76 & 3.8 & 3.10 & 6.90 & 2 \\
Museo Parroquial Uzhcurrumi & 43.5 & 2.18 & 3 & 5.18 & 6 \\
Parque Central Uzhcurrumi & 40 & 2 & 3.6 & 5.60 & 5 \\
Petroglifos & 41 & 2.05 & 2.7 & 4.75 & 10 \\
Puente Histórico & 36.5 & 1.83 & 2.7 & 4.53 & 12 \\
Balneario de Quera & 55 & 2.75 & 3.15 & 5.90 & 4 \\
Balneario Chillayacu & 83.5 & 4.18 & 3 & 7.18 & 1 \\
Cascada Porotillo & 44 & 2,2 & 2.6 & 4.80 & 9 \\
Artesanía & 32 & 1.6 & 2,2 & 3.80 & 14 \\
Fiestas Religiosas & 35.5 & 1.78 & 2.6 & 4.38 & 13 \\
Fiestas Parroquiales & 41 & 2.05 & 2.6 & 4.65 & 11 \\
\hline
\end{tabular}

Fuente: Elaboración Propia a partir de Franco (2009)

Se puede observar que el recurso con mayor potencial turístico es el Balneario de Chillayacu, debido a que es el atractivo con mejor acceso y que cuenta con la mejor infraestructura, lo que permite mayores posibilidades de éxito, para su potencializarían. Es evidente, que tiene la ventaja de que al ser extenso, tiene mayor relevancia por sus criterios intrínsecos. Los criterios extrínsecos que lo definen son altos y como era previsible, tiene gran potencial para desarrollarse turísticamente, pues es el recurso que más visitantes atrae dentro de la Parroquia. La evaluación aplicada permite visualizar que las manifestaciones culturales de la parroquia son frágiles, por eso el recurso cultural "artesanías" ha quedado hasta el final de la lista, pues no depende solo de su potencialidad, sino además de su estado de conservación, participación comunitaria y la promoción. Un análisis general de los resultados obtenidos permite observar que, cuando se realiza directamente la sumatoria de todos los criterios normalizados, pero sin aplicar ningún criterio de ponderación, son los criterios extrínsecos los que ejercen una influencia determinante en la evaluación. En este contexto tienden a prevalecer en los primeros lugares los sitios turísticos, como el Balneario de Chillayacu, La Tarabita de Leo y Las Cavernas de Chillayacu.

La evaluación multicriterio permite valorar las variables distancia y tiempo, puesto que éstas son consideradas de gran relevancia en el desplazamiento para el disfrute de los recursos; sin embargo, si bien ejercen influencia, no son las determinantes en la evaluación final ya que recursos como "Las Artesanías" y el "El Puente Histórico", se ubican en las últimas posiciones del listado y su localización es relativamente cercana. En términos del interés natural los recursos más significativos son: Balneario de Quera y la Cascada Porotillo. No obstante, su belleza natural y paisajística, no es suficiente para que se ubiquen en los primeros lugares; la falta de equipamiento, instalaciones y seguridad devalúan su posición. En síntesis, la jerarquización realizada permitió apreciar que los sitios como el Balneario de Chillayacu, La Tarabita de Leo y el Balneario de Quera, son los lugares pertinentes para emprender la estructuración de una oferta en el marco de un proceso de planificación estratégica de desarrollo turístico sustentable.

\section{DISCUSIÓN}

La Parroquia Uzhcurrumi, cuenta con los atractivos turísticos para realizar actividades de turismo de aventura, turismo rural y ecoturismo; dado que los recursos naturales son viables para tales actividades debido a su estado casi intacto por la mano del hombre, y aun cuando actualmente posee instalaciones para recibir al turismo, el inventario 
refleja que se puede impulsar en mayor medida la actividad turística, siempre hacia una visión sustentable.

Cabe citar que (Franco, Osorio, Navabernal, \& Regil, 2009), manifiestan que la falta de visión en la propuesta de un desarrollo turístico, afecta a corto o largo plazo el destino de los recursos y a las comunidades, lo cual puede ser previsto si se realiza un adecuado inventario de recursos turísticos, y a partir de ello se elaboran propuestas de desarrollo turístico que permita el aprovechamiento sustentable de éstos recursos y genere beneficios a las comunidades.

La adecuada selección de un inventario de recursos turísticos permitirá diseñar propuestas de desarrollo de productos turísticos, encaminados a generar el mínimo impacto en la comunidad y una maximización en la experiencia del turista. La elaboración de un adecuado inventario y la evaluación multicriterio se convierten en una guía de acción que permiten identificar la oferta turística presente y las acciones que faltan por realizar en el destino.

Finalmente, la evaluación de los recursos turísticos es una herramienta valiosa para orientar la toma de decisiones en los procesos de planificación y desarrollo turísticos. A partir de la identificación de los recursos de mejor posi- ción, como son el Balneario de Chillayacu, La Tarabita de Leo y Las Cavernas de Chillayacu, se puede elaborar la propuesta de creación de productos alternativos encaminados a prácticas ecoturísticas y turismo de aventura. La evaluación realizada ha permitido distinguir claramente los recursos con mayores atributos para su uso recreativo a partir de sus características propias. No obstante, es importante mencionar que la evaluación realizada tiene como limitante la falta de consideración respecto a los actores sociales vinculados con los recursos.

\section{REFERENCIAS}

Barba, S. \& Pomerol, J. (1997). Decisiones Multicriterio. Fundamentos teóricos y utilización práctica. Alcalá de Henares: Colección Economía.

Blanco, P., Vázquez, V., Reyes, J. \& Genet, M. (2015). Inventario de recursos turísticos como base para la planificación territorial en la zona altiplano de San Luis de Potosí, México. Cuadernos de Turismo, 17-42.

Bote, V. (2002). Planificación económica del turismo. México: Trillas.

Cárdenas, F. (2012). Proyectosturísticos: Localización e inversión. México: Tillas.

Enríquez, M. (2010). Evaluación multicriterio de los recur- 
sos turísticos del Parque Estatal Sierra Nanchititla, Estado de México. El Periplo Sustentable, 7-35.

Font, M., Cuétara, L. \& Castanedo, Y. (2013). Inventario de actractivos para el desarrollo turístico Local del Municipio Martí. Revista Avanzada Científica, 1-21.

Franco, S., Osorio, M., Navabernal, G. \& Regil, H. (2009). Evaluación multicriterio de los recursos turísticos. Parque Nacional Nevado de Toluca- México. Scielo, 208-226.

Godfrey, K. \& Clarke, J. (2000). The Tourism development handbook. London: Continuum.

Gutiérrez, J., Castillo, R., Castañeda ,J. \& Sánchez, J. (1998). Recursos Naturales y Turismo. México: Limusa.

Herrera , G., Crespo, G., Zambrano, D. \& Cadena, P. (2014). La propuesta metodológica para la valoración y sistematización de Recursos Turísticos. Revista Retos, 125-142.

Laguna, Y.,\& Bravo, N. (2001). La potencialidad turística del medio natural en el LIC de Las Sierras Ibéricas Riojanas medianteevaluación multicriterio. Zubía Monográfico, 227-240.

Malczewski, J. (2000). On the Use of Weighted Linear Combination Method in GIS: Common and best practice approaches. Transactions in GIS, 5-22.

Mikery, M.,\& Pérez, A. (2017). Métodos para el análisis del potencial turístico del territorio rural. Revista de Ciencias Agrícolas, 1729-1740.

MINTUR. (2017). Guía metodológica para la jerarquización de atractivos y generación de espacios turísticos del Ecuador . Quito: Textoland.

Morales, J. (2013). Inventario turístico municipio de Querétaro. México: Congreso de Investigación Turística.

Ocaña, C. \& Galacho , F. (2002). Un modelo de Aplicación de SIG y Evaluación Multicriterio, al Análisis de las capacidades del Territorio en relación a Funciones Turísticas. Turismo y Técnologías de la Información y las Comunicaciones, 235-253.

Oscos, J. (1990). Metodología para la elaboración de inventarios de atractivos turísticos. Obtenido de Facultad de Turismo de la UAEM.

SECTUR. (2004). Cómo desarrollar un proyecto ecoturístico. México: Primerts.

SECTUR. (2004). Planeación y gestión del desarrollo turístico. México: SECTUR.

Vega, J. (2005). Inventario y evaluación de los recursos naturales y culturales del Parque Nacional Iztaccihuatl-Popocatépetl. . México: Facultad de Turismo de la UAEM.

Zamorano, F. (2002). Turismo alternativo. Servicios turísticos diferenciados. México: Trillas. 